\title{
Equivalencia entre medidas eléctricas y difracción de rayos $X$ en la formación de fases cristalinas de pastas de cemento
}

\author{
E. MENÉNDEZ ${ }^{1}$ AND J. DE FRUTOS ${ }^{2}$ \\ ${ }^{1}$ IETCC-CSIC C/ Serrano Galvache, 4 - 28033 Madrid (Spain). emm@ietcc.csic.es \\ ${ }^{2}$ POEMMA R\&D Group ETSIT-UPM. Ciudad Universitaria s/n. 28040 Madrid
}

\begin{abstract}
En este trabajo se hace un estudio comparado de los procesos de hidratación en edades muy tempranas, 20 primeras horas, entre una pasta de cemento convencional y su equivalente con una sustitución de escorias de aproximadamente el $12 \%$. El estudio se realiza mediante calorimetría semiadibática, espectroscopía de impedancia y difracción de rayos X. Se pone de manifiesto que haciendo uso de la espectroscopía de impedancia se puede determinar el estado de cristalización del material con y sin adiciones, permitiendo determinar el tiempo en el que se inician los diferentes procesos de hidratación en el mismo.
\end{abstract}

Palabras clave: Impedancia Eléctrica, hidratación cemento, escorias, análisis no destructivo

Equivalence between electrical measurements and $\mathrm{X}$ ray difraction in the formation of crystalline phases of cement paste

In this paper a comparative study of the hydration process in a very early age, first 20 hours, between a conventional cement paste and its equivalent with a replacement of slag of about $12 \%$, is done. The study was undertaken through semiadibatic calorimetry, electrical impedance spectroscopy and X-ray diffraction. It shows that using electrical impedance spectroscopy we can determine the state of crystallization of the cement with and without additions, thereby determining the time at which the different processes are initiated in the hydration

Keywords: Electrical impedance, hydration cement, slag, non-destructive testing

\section{INTRODUCCIÓN}

En el cemento y sus derivados, el uso de la impedancia eléctrica, y en especial de la espectroscopía de impedancia se inició por Bares et al. (1), a imitación del estudio que se realizaba en materiales cerámicos. A medida que mejoraba la instrumentación y se pudo ampliar el rango de frecuencia, se aplicó esa técnica para el estudio de pastas de cemento en un amplio rango de frecuencias (20-110 $\mathrm{MHz}(2))$ con resultados dudosos hasta que a comienzos de los noventa esta técnica empezó a ser utilizada de manera habitual para estudiar las pastas de cemento, analizándose pastas de cemento Portland con relación agua cemento 0,27 a tres edades de hidratación (1, 10 y 100 días) (3). Posteriormente, se estudiaron estos fenómenos con diferentes formas de procesado de material y condiciones de estudio $(4,5,6)$, y se determinaron relaciones entre los espectros de impedancia (7) y las características microestructurales $(8,9)$ de los materiales. Más tarde se analizó el comportamiento de diferentes pastas en función del procedimiento de fabricación, de las condiciones de curado y del tipo de contacto eléctrico $(10,11)$.

Algunos de los experimentos antes mencionados se dedicaron a estudiar las relaciones entre la microestructura porosa y el comportamiento eléctrico de pastas de cemento (7-10), y otros a investigar la evolución de las propiedades eléctricas con el estado de hidratación o el grado y tipo de deterioro del material (8-16). Hubo quien se centró en el estudio de la hidratación de pastas de cemento mediante la espectroscopía de impedancia (18), asumiendo que la pasta de cemento es conductor eléctrico debido a su porosidad y a la conectividad presente, además, al tratarse de un material hidratado, hay una fase acuosa en el material que contiene iones móviles tales como $\mathrm{Na}^{+}, \mathrm{K}^{+}$y $\mathrm{OH}^{-}(19,20)$ y que se puede ver acentuado por procesos de degradación externos como los ciclos hielo-deshielo. En general, en los últimos años, esta técnica se ha utilizado en los cementos para estudiar la evolución microestructural y, en particular, la estructura de poro $(2-10,20)$. Se han propuesto varios modelos de circuitos equivalentes para estimar los parámetros físicos significativos de las respuestas eléctricas en función de la estructura, distribución y tamaño de poro, junto con el grado de hidratación $(21,11-25)$ siempre para tiempos superiores a las 24 horas, en los que la estabilidad del material permite el análisis de los arcos de impedancia.

Un elemento que determinará en gran medida la posterior microestructura del material, es el proceso de hidratación inicial. Por su parte, la utilización de adiciones de subproductos industriales, implica una mayor complejidad de estos procesos de hidratación, modificándose tanto el tiempo de fraguado como algunos de los productos de hidratación formados. Así mismo, las reacciones de hidratación tienen una elevada entalpía, generado un importante calor durante 
el proceso, por lo que la medida mediante calorimetría se ha utilizado tradicionalmente para el análisis de la evolución de la hidratación en los distintos tipos de cementos (26). El proceso de hidratación inicial es determinante en el estado final de las propiedades mecánicas del material, y de su potencial durabilidad. De nuevo, la espectroscopía de impedancia eléctrica se presenta como una herramienta potente para discriminar este tipo de fenómenos de manera similar a como se ha utilizado en materiales cerámicos con fases o adiciones diferentes $(27,28)$

En la hidratación del cemento sin adición se forman, como productos principales, los silicatos cálcicos hidratados (C-S$\mathrm{H})$, que actúa como matriz del cemento, y el hidróxido cálcico (CH) (portlandita), que se forma tanto en la matriz como en el interior de poros. Además, la reacción de los aluminatos y ferritoaluminatos, en presencia de yeso, da lugar a la formación de ettringita $\left(\mathrm{C}_{6} \mathrm{AS}_{3} \mathrm{H}_{32}\right)$ que, en una segunda etapa y en defecto de azufre, puede reaccionar con el aluminato para formar monosulfoaluminato $3 \mathrm{C}_{4} \mathrm{ASH}_{12}$, apareciendo estos compuestos tanto en la matriz como en las zonas porosas del material $(19,29)$. Por su parte, la hidratación de los cementos con adiciones requiere el estudio de los mecanismos de hidratación tanto del cemento Pórtland, como de cada una de las adiciones, así como el proceso de interacción. En el caso de los cementos con escorias de horno alto, además de los productos de hidratación del cemento, se forma una nueva fase del tipo hidro-calcita (30). Por otra parte, hay que tener en cuenta que cuando se usan adiciones, la cinética de reacción se modifica con relación al cemento.

Los procesos de hidratación son fuertemente exotérmicos, fundamentalmente en las primeras etapas, por lo que el análisis mediante calorimetría semi-adiabática constituye un indicativo del inicio y evolución de ésta, además de caracterizar el comportamiento térmico del material en las etapas iníciales (26).

Para mejorar el conocimiento del material en esta etapa, hemos desarrollado un nuevo procedimiento de estudio de las reacciones iniciales de hidratación en estos materiales, basado en la medida de la impedancia eléctrica a diferentes frecuencias y en función del tiempo (31). La utilización de la espectroscopía de impedancia eléctrica, exige la determinación de los arcos de impedancia en rangos de alta y baja frecuencia, lo que requiere tiempos de medida superiores a los 30 minutos para cada dato, con lo que muchos de los estados iniciales e intermedios se pierden, y la medida se obtiene en una etapa de cambios muy notables en el material, lo que limita mucho su validez. Como hemos comentado anteriormente, el proceso de hidratación en estos materiales, conlleva cambios notables en la presencia de iones en disolución. La mayor o menor presencia de iones, unido a la movilidad de cada uno de ellos, y a la posibilidad de uniones y enlaces dará lugar a cambios muy significativos en la respuesta eléctrica del material. La medida de esta respuesta eléctrica en diferentes condiciones y frecuencias de trabajo y su posterior relación con la presencia de iones y en consecuencia con las reacciones químicas que están teniendo lugar en el material ha de proporcionar una herramienta de trabajo versátil, rápida y no destructiva para el estudio de estos fenómenos (31).

Para estos mismos materiales, determinaremos tanto los calores de hidratación como la evolución de la difracción de rayos $\mathrm{x}$, para disponer de elementos comparativos que nos permitan relacionar la respuesta eléctrica con los procesos que tienen lugar en el material. Así, dispondremos de una técnica que de una manera rápida y en medidas in situ, permita determinar el estado de hidratación del material.

\section{EXPERIMENTAL}

Se han diseñado y analizado dos pastas de cemento con las características y composición química que se indican en la tabla 1. La pasta P-I que se utilizará como referencia, y la pasta P-S que corresponda a la pasta con una adición del 12,1 $\%$ de escoria de horno alto. Se incluye también en la tabla la composición química de las escorias utilizadas como adicción. Las diferentes composiciones de los cementos influyen tanto en el desarrollo de la hidratación, como en la microestructura de los productos formados durante la hidratación.

El análisis del calor desarrollado durante las etapas iniciales de la hidratación de los cementos permite ver la evolución de ésta, además de cuantificar el calor desarrollado. El calor de hidratación es una función de la naturaleza del

\begin{tabular}{|c|c|c|c|c|c|c|c|c|c|c|c|c|c|c|c|c|c|c|c|}
\hline \multicolumn{20}{|c|}{ Composición química de las pastas con y sin adicción, y de la escoria } \\
\hline & & 吕 & $\frac{0^{m}}{z^{-}}$ & $\stackrel{0}{\infty}_{\mathcal{O}^{-}}$ & $\begin{array}{l}\text { О } \\
\text { J్ }\end{array}$ & $\begin{array}{l}\text { Oo } \\
\sum\end{array}$ & ర) $^{m}$ & $\begin{array}{l}O^{N} \\
\tilde{Z}^{\prime \prime}\end{array}$ & $\begin{array}{l}\mathbf{O}^{\prime} \\
\mathbf{r}^{\prime}\end{array}$ & $\stackrel{\Omega}{日}^{N}$ & $\begin{array}{l}O^{10} \\
\alpha^{n}\end{array}$ & نه: & $\dot{ப}$ & స్ & $\begin{array}{l}0^{N} \\
j \\
+ \\
0 \\
\sum_{0}^{+0} \\
0 \\
0 \\
\tilde{J}\end{array}$ & 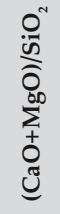 & 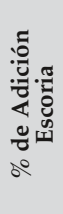 & 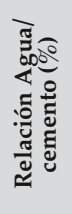 & 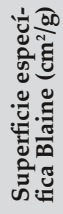 \\
\hline \multirow{2}{*}{ CEMENTO } & P-I & 20,05 & 5,19 & 2,2 & 62,04 & 3,61 & 2,83 & 0,3 & 0,86 & 0,24 & 0,14 & 2,52 & 0,03 & 100 & & & 0 & 27,86 & 4101 \\
\hline & P-S & 22 & 6,18 & 2,22 & 59,2 & 4,29 & 2,34 & 0,28 & 0,81 & 0,32 & 0,12 & 2,2 & 0,04 & 100 & & & 12,1 & 28,22 & 4218 \\
\hline \multicolumn{2}{|c|}{ ESCORIA } & 38,42 & 11,31 & 1,54 & 40,76 & 6,54 & 0,24 & - & 0,32 & 0,71 & 0,02 & - & 0,14 & 100 & 85,3 & 1,23 & & & \\
\hline
\end{tabular}


cemento $\mathrm{y}$, generalmente, está comprendido entre $10^{\circ} \mathrm{C}$ y $50^{\circ} \mathrm{C}$. Para medirlo, el material cementoso se introduce, después de amasarlo, en un recipiente Dewar con un tapón térmicamente aislante, en cuya parte central se introduce un termopar, registrándose el calor producido a intervalos definidos de tiempo. La temperatura de hidratación se registra mediante un voltímetro. En este caso se midió la temperatura cada 5 segundos, durante un periodo total de 48 horas. Posteriormente se tratan los datos con el objetivo de determinar el proceso de variación diferencial del calor específico. Con esto se pretende resaltar los efectos y cambios del proceso en función del tiempo.

Para la realización de las medidas dieléctricas, se ha desarrollado un portamuestras específico (31) que permite obtener la respuesta del material desde las primeras etapas de la hidratación.

En este portamuestras se disponen dos electrodos separados por material aislante en una región que estará cubierta de la mezcla previa a la hidratación. Posteriormente se introduce el agua y, mediante un agitador de alta velocidad (1000-5000 rpm), se procede a uniformar la mezcla. Esta disposición permite realizar la toma de datos desde antes de iniciarse el proceso de hidratación. En la Figura 1 se muestra un esquema del portamuestras y del sistema de medida.

Las medidas de impedancia se han realizado con un analizador solartron 1260A Impedance/Gain-phase con el módulo de medida dieléctrica 1296A, lo que permite el análisis en un rango de frecuencias de $10 \mu \mathrm{Hz}$ a $10 \mathrm{MHz}$, de impedancias $100 \mathrm{ohm}$ a $100 \mathrm{Tohm}$, con una resolución en frecuencia de 1 en $65,000,000$ y con posibilidad de aplicar bias DC en el rango de $\pm 40,95 \mathrm{~V}$. Para el tratamiento de los resultados se ha utilizado software específico desarrollado por los autores y el paquete de software de solartron ZView. Se adquirieron los valores de respuesta eléctrica del material con ciclos continuos de datos en el rango de $10^{6} \mathrm{~Hz}$ hasta $10^{-3}$ $\mathrm{Hz}$ en barrido logarítmico con dos puntos por década. El inconveniente de este proceso, es que cada ciclo precisa de al menos 30 minutos para realizarse, con lo que la información sobre la respuesta inicial de la hidratación de los cementos no es de gran utilidad, pero sí nos ayudará a diferenciar procesos químicos que tienen lugar y la posibilidad de asociar éstos con la respuesta eléctrica obtenida.

Con el objetivo de determinar el tipo de reacción química que tiene lugar en el material, se ha realizado un estudio de la evolución de la respuesta de DRX durante el proceso de hidratación. Las medidas de difracción de rayos $X$ se han realizado con un equipo Bruker D8 Advance con ánodo de $\mathrm{Cu}$ de 2,2 kW, generador de rayos $\mathrm{X}$ con potencia $3 \mathrm{~kW}$, tensión $20-60 \mathrm{~kW}$ y corriente $5-80 \mathrm{~mA}$, goniómetro theta / theta y detector ultra rápido Lynxeye.

Las medidas se han realizado secuencialmente en continuo durante la hidratación durante las primeras 12 horas de hidratación de los cementos. La duración de cada barrido secuencial es de 10 minutos, manteniéndose un ambiente de humedad controlada superior al $80 \%$.

\section{RESULTADOS Y DISCUSIÓN.}

En la figura 2 se representa tanto el valor del calor de hidratación como su diferencial. Puede observarse diferentes comportamientos para cada uno de ellos, lo que a grandes rasgos coincide con los tiempos en los que tiene lugar la fase intermedia del proceso de hidratación que es cuando se

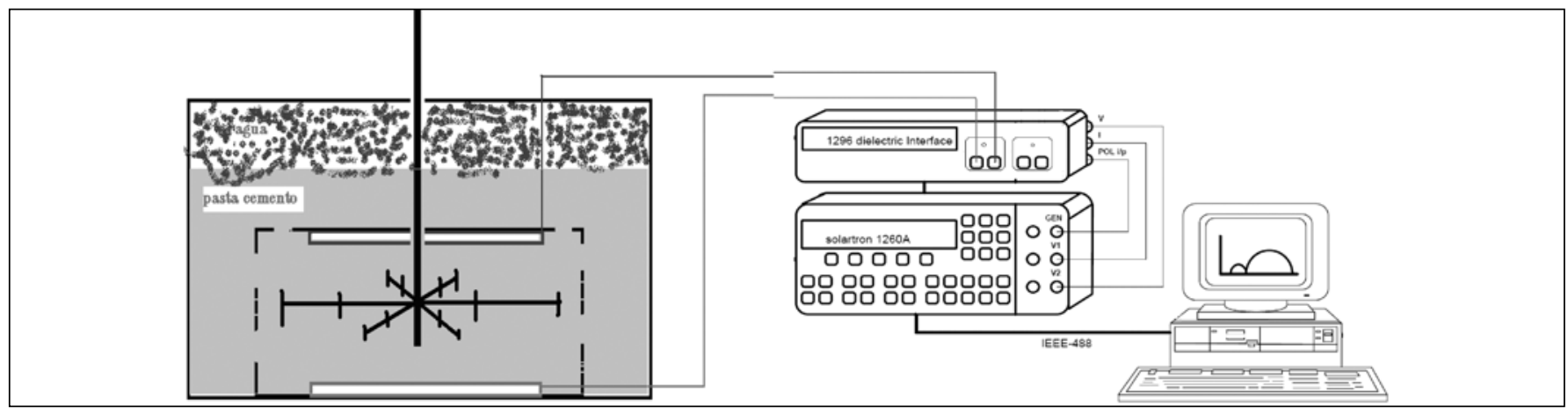

Figura 1.Esquema del sistema experimental desarrollado para el estudio de la respuesta de impedancia en las primeras etapas de hidratación
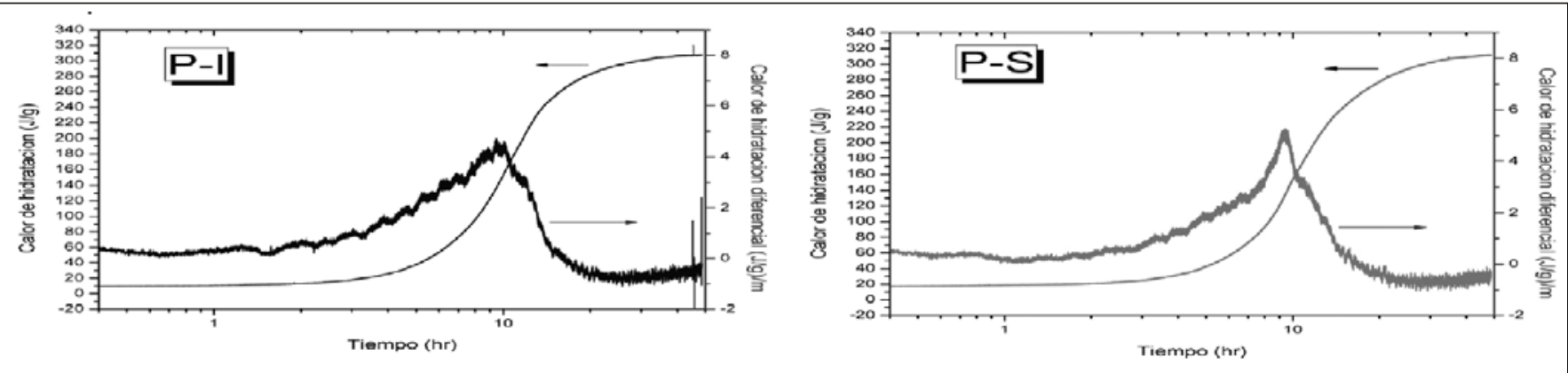

Figura 2. Evolución con el tiempo del calor de hidratación para las dos composiciones 


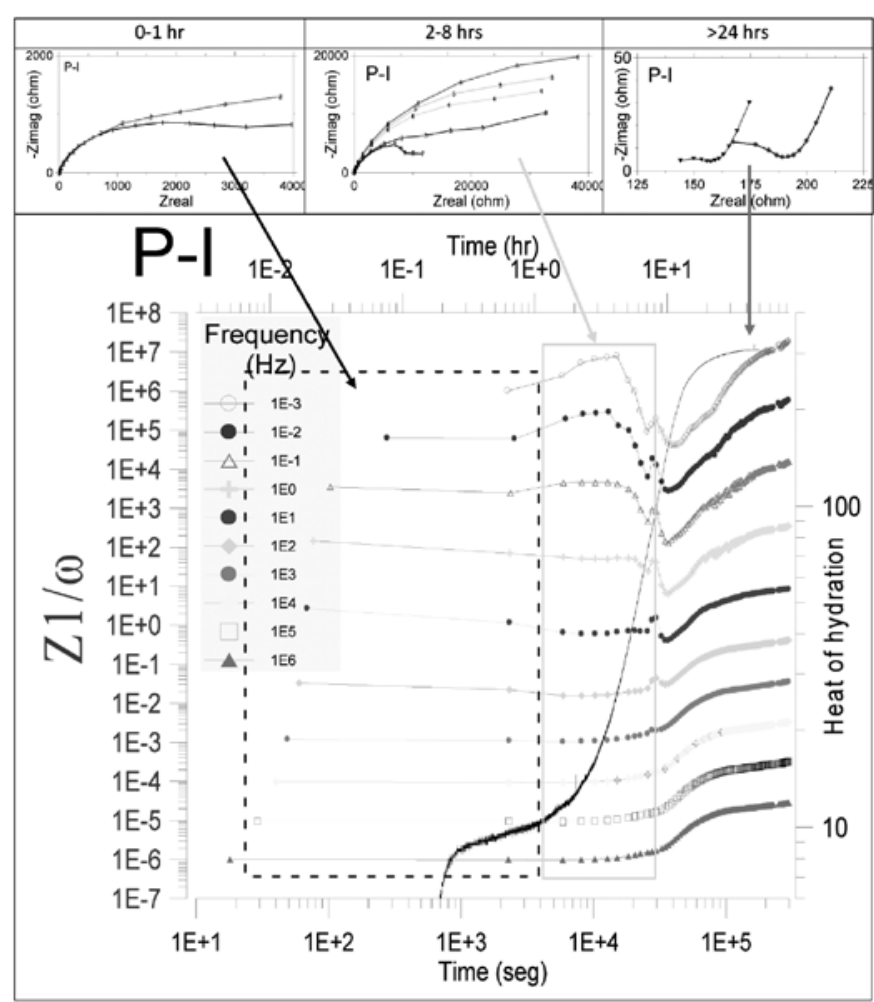

Figura 3. Evolución de la impedancia real normalizada a la frecuencia, en función de tiempo, y para diferentes frecuencias de análisis, y curva del calor de hidratación para la pasta P-I, libera mayor cantidad de calor en el material. En el resto del proceso no se observan comportamientos significativamente diferenciados que permitan relacionarlos con los procesos que van teniendo lugar en el interior del material.

Como se ha indicado anteriormente, la respuesta eléctrica de las pastas se modifica notablemente debido a la diferente composición de éstas. En las figuras 3 y 4, de acuerdo con el modelo clásico de espectroscopía de impedancias, se representa $Z^{\prime \prime}\left(Z^{\prime}\right)$ para cada una de las muestras elegidas para diferentes etapas del proceso de hidratación. La falta de arcos bien definidos, la dificultad para determinar tiempos de relajación, y especialmente el periodo de tiempo necesario para poder completar cada ciclo (aproximadamente 2000 segundos) hace que sea difícil determinar a partir de esta información los procesos relacionados con la hidratación. Para conseguir la información que buscamos, representamos posteriormente la evolución de un parámetro eléctrico, $Z^{\prime}$, normalizándolo a la frecuencia para visualizar mejor los fenómenos, en función del tiempo y para un amplio espectro de frecuencias. Superpuesto a estos valores, se ha incluido una curva correspondiente al calor de hidratación en el material puesto que, como se ha comentado con anterioridad, se han utilizado las medidas de calor específico para entender las reacciones que tienen lugar en este proceso.

De las figuras 3 y 4, se desprende un comportamiento claramente diferenciado para cada una de las pastas y con respuestas distintas para cada frecuencia. Por su parte, el proceso de hidratación determinado por calorimetría, es similar al de la respuesta eléctrica a altas frecuencias. Sin embargo, en el rango de bajas frecuencias se observan alteraciones en la respuesta de las pastas. La medida de la

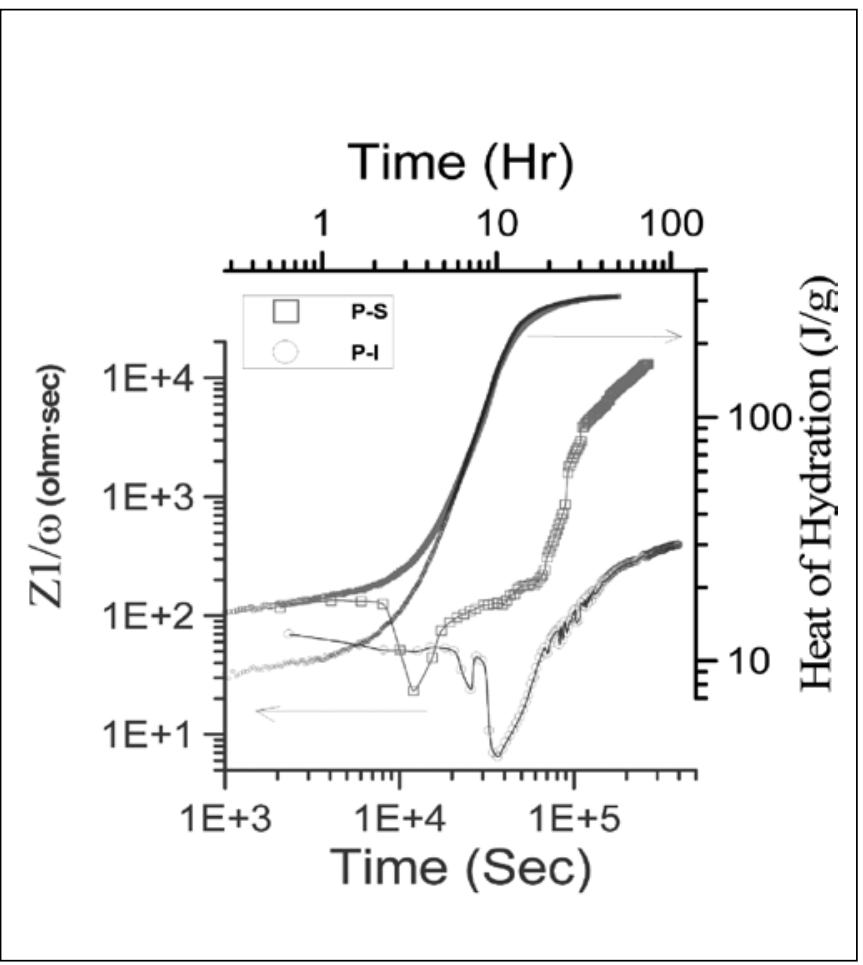

Figura 5. Evolución comparada con el tiempo de la impedancia real a $1 \mathrm{~Hz}$ de frecuencia, para las dos pastas estudiadas.
Figura 4. Evolución de la impedancia real normalizada a la frecuencia, en función de tiempo, y para diferentes frecuencias de análisis, y curva del calor de hidratación para la pasta P-S.

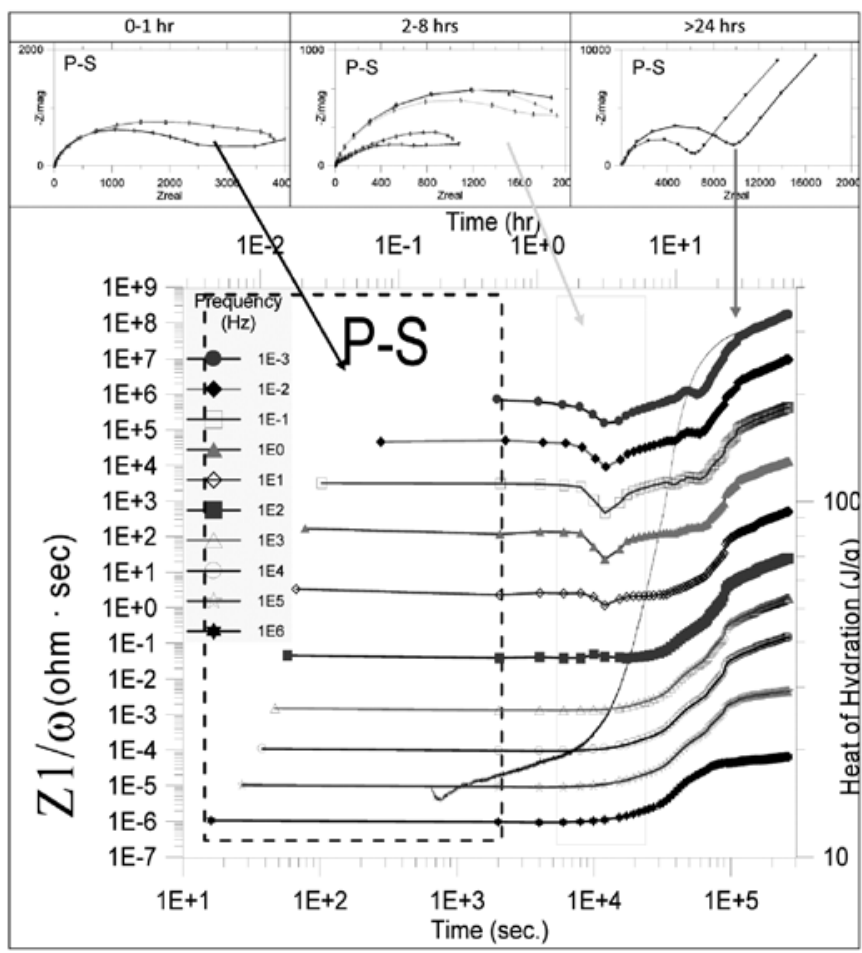


impedancia, y especialmente su frecuencia de trabajo, es una herramienta potente para determinar los fenómenos y procesos químicos que tienen lugar en estos materiales en las etapas iniciales de hidratación. Cada rango de frecuencias proporciona informaciones diferentes. Así las altas frecuencias permiten determinar el comportamiento general del material de forma similar a como lo hace el calor de hidratación, aunque con un pequeño desfase en tiempo respecto a éste. Por su parte, los estudios con bajas frecuencias ponen claramente de manifiesto la presencia de diferentes fenómenos y reacciones que van teniendo lugar en el proceso de hidratación.

La mayor limitación que nos encontramos, es que para hacer estudios a bajas frecuencias, cada medida exige tiempos largos que puede hacer que algunos de los fenómenos que tienen lugar en tiempos cortos no se lleguen a visualizar. Con frecuencias superiores al $\mathrm{Hz}$, los resultados que se obtienen son suficientemente discriminadores de los fenómenos que tienen lugar, y requieren tiempos de medida suficientemente cortos como para que no se escapen fenómenos en el proceso de medida.

Para hacer un primer análisis de los procesos estudiados, se comparan los resultados para las dos pastas ensayadas obtenidos con los valores de hidratación y los valores de impedancia determinados a $1 \mathrm{~Hz}$ y en función del tiempo. Posteriormente, compararemos estos resultados con los de Difracción de Rayos X.

Los calores de hidratación presentan un comportamiento muy similar, por lo que su análisis es difícil de utilizar para poder inferir el tipo de fenómenos que están teniendo lugar. Para tratar de completar información a partir de estos datos, se ha determinado el valor diferencial de los calores de hidratación, que recogen en la figura 5 para las dos pastas.
En la Figura 5 se recogen los datos correspondientes a la respuesta eléctrica a $1 \mathrm{~Hz}$, comparada con el calor de hidratación asociado para cada cemento. Del análisis de los resultados anteriores, se desprende que la respuesta eléctrica presenta cambios muy significativos entre las diferentes pastas estudiadas desde tiempos muy cortos. Podemos observar con cuadrados, la respuesta del material P-I, y con círculos la del P-S. En ambos casos se inicia una disminución de la impedancia en aproximadamente las dos horas, pero mientras que en el caso del P-I el proceso muestra un continuo llegando a un mínimo, y posteriormente volviendo a crecer, el P-S presenta una primera disminución de la impedancia en el mismo rango que P-I pero con una variación muy pequeña, con un posterior repunte para iniciar una brusca disminución en dos etapas que se inicia con un retraso de al menos 3 horas, y que se manifiesta de forma muy brusca con un retraso de 7 horas.

La liberación de iones de la pasta sin adiciones o de la pasta con escorias permite una mayor presencia de portadores, y en consecuencia la fuerte disminución de la impedancia del material. Dependiendo de las reacciones químicas que tengan lugar en el mismo, para las diferentes composiciones las reacciones tienen lugar a diferentes tiempos y con duraciones y recorridos diferentes. Los retardos observados en los procesos de hidratación respecto al P-I, son debidos a la naturaleza de las adiciones.

En la hidratación de la pasta P-S, se identifica una primera etapa con una presencia importante de iones (donde hay un aumento de hidróxido de calcio en disolución, al liberarse el Ca de la superficie del C3S con un aumento del pH). Posteriormente, entre 2,5 y 4 horas se produce una reducción

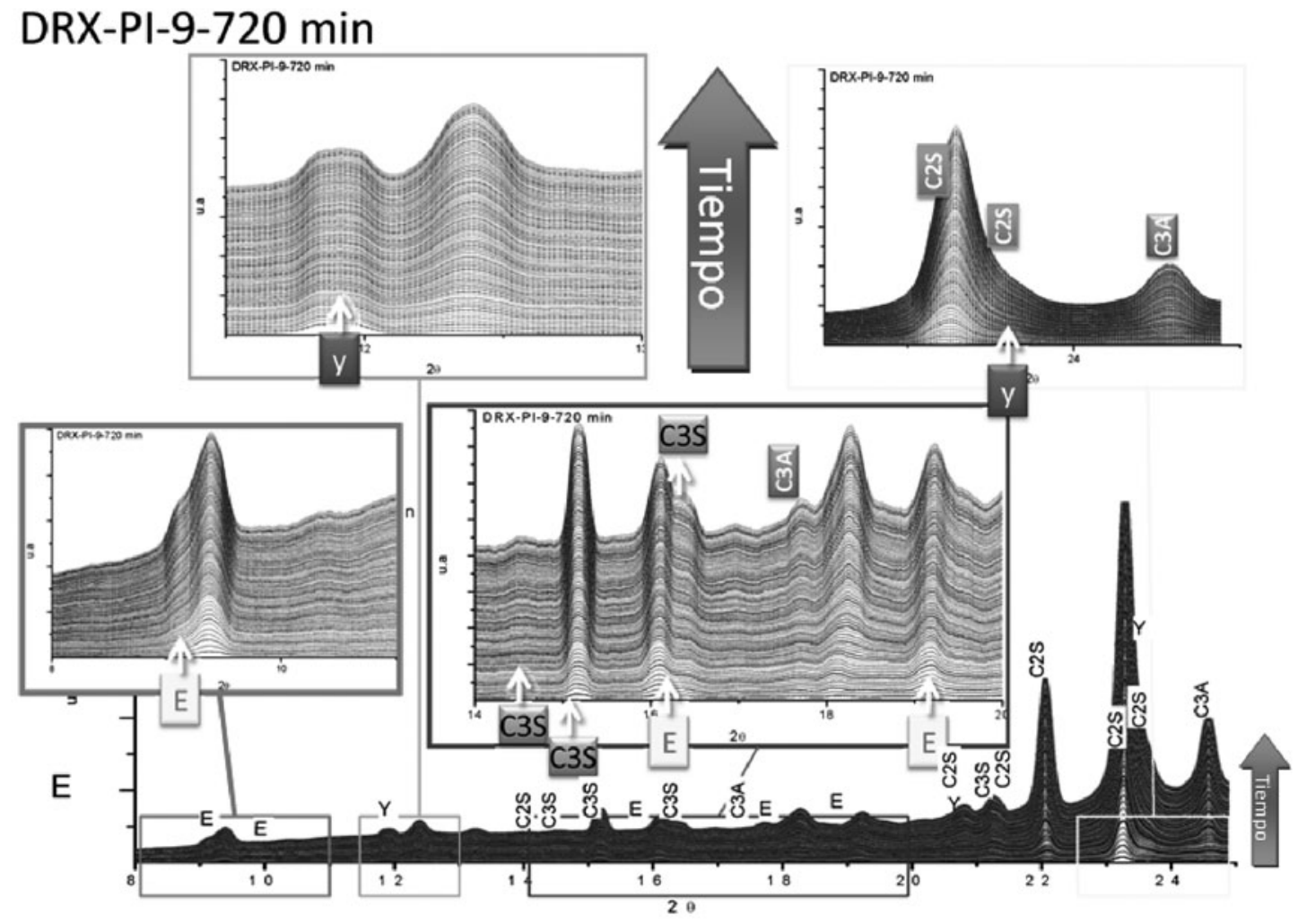

Figura 6. Evolución del espectro de DRX de la muestra P-I durante las doce primeras horas del proceso de hidratación. 

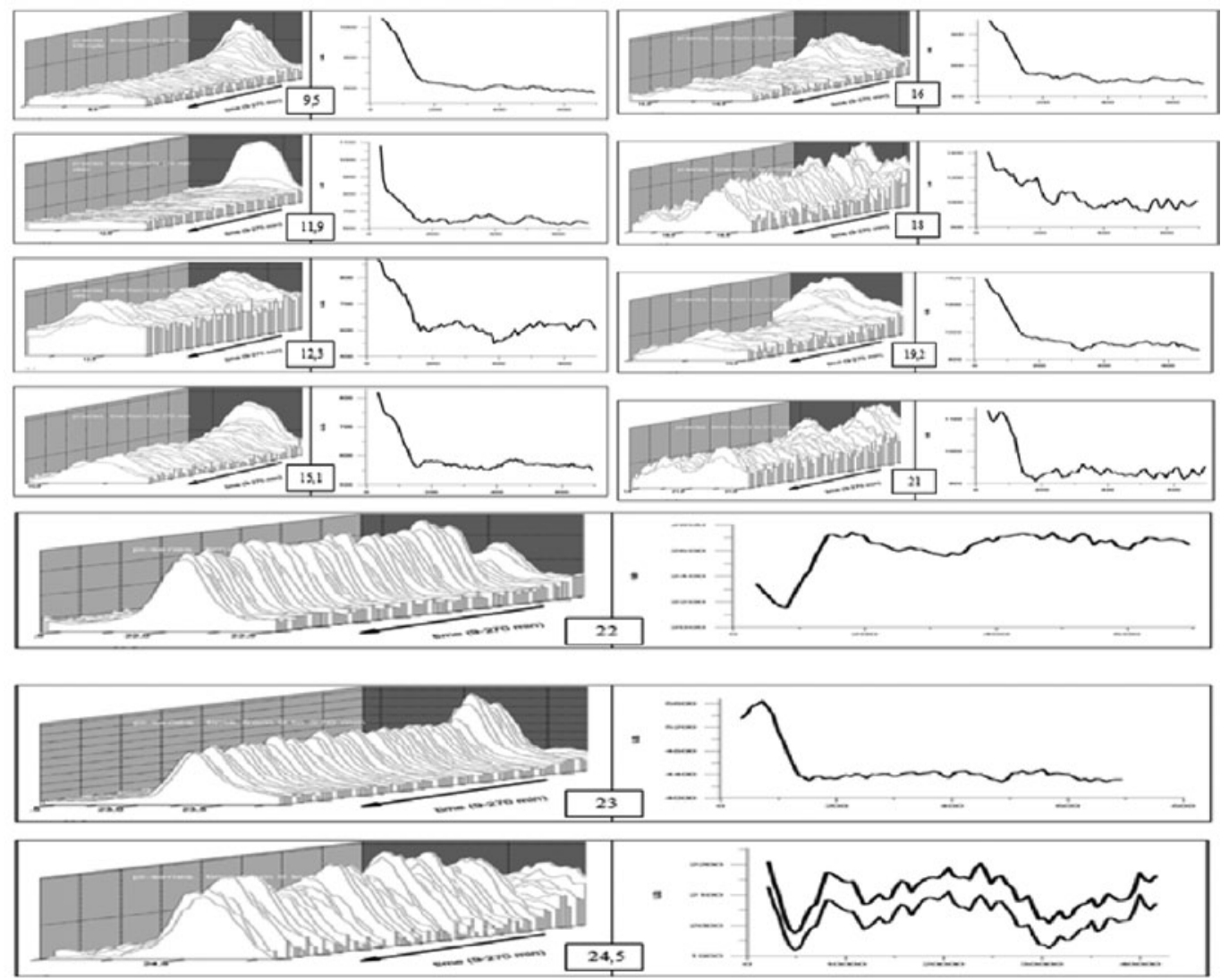

Figura 7. Evolución de diferentes picos de DRX de la muestra P-I durante las doce primeras horas del proceso de hidratación. Se indica el valor central del pico analizado

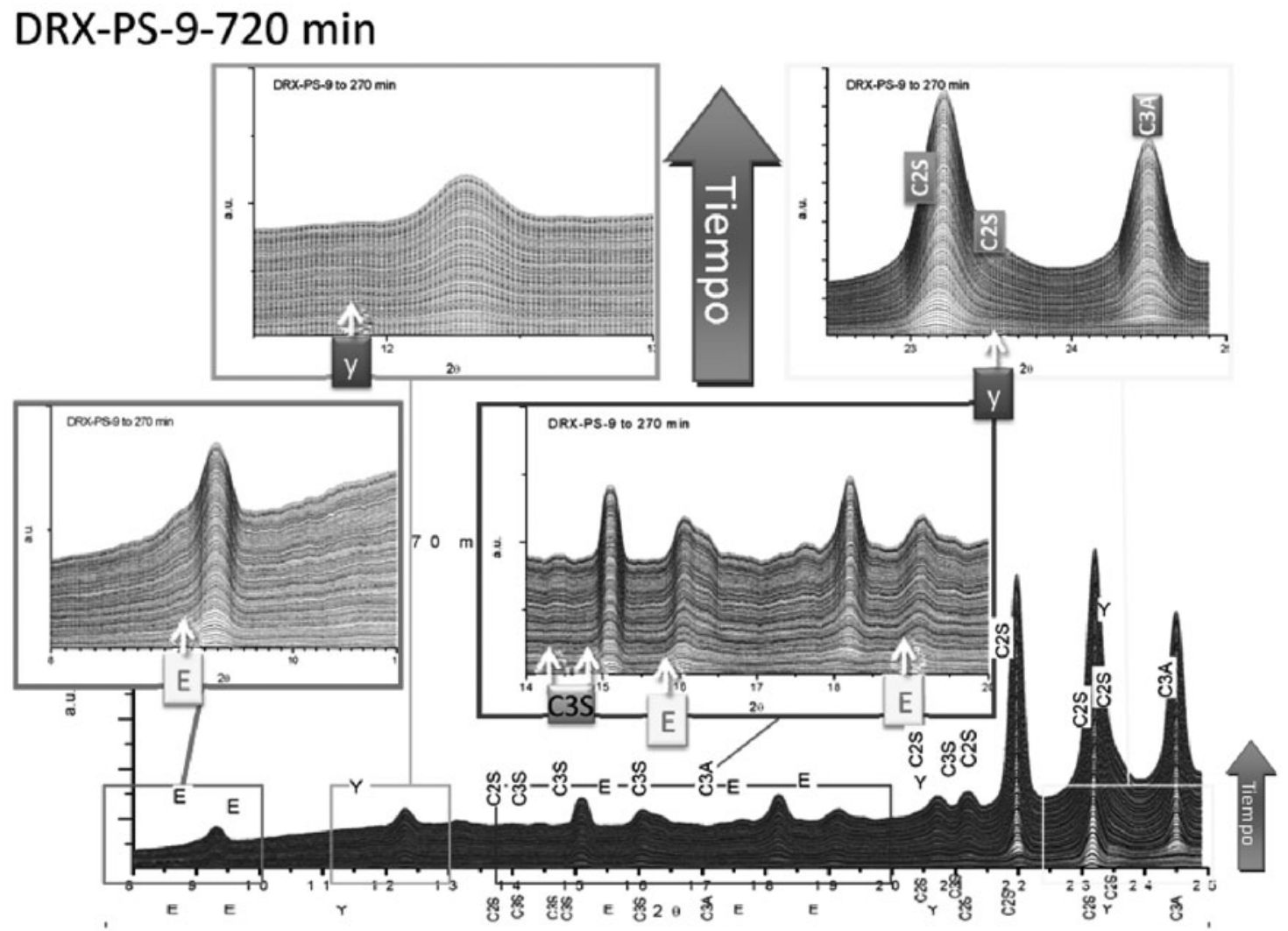

Figura 8. Evolución del espectro de DRX de la muestra P-s durante las doce primeras horas del proceso de hidratación. 
de la presencia de portadores, que se asocia con el periodo inactivo (en el que los granos de clinker se recubren con productos de hidratación por lo que la reacción queda latente) esta etapa se prolonga durante el tiempo que tarda el agua en penetrar en los granos de clinker y en recubrir y activas las escorias añadidas como adicción. Tras este periodo se observa un aumento gradual de portadores, debido a la liberación de iones desde las partículas, cuando el agua penetra en el recubrimiento en un proceso de difusión controlada. A partir de esta etapa se produce una hidratación continuada con la reacción de los compuestos menos reactivos, como el C2S.

Por su parte, en la hidratación de la pasta P-I se observa una ligera disminución de la conductividad, lo que sugiere un ligero aumento de la presencia de portadores entre 2,5 y 3,5 horas. Esta suave variación se puede asociar con un doble proceso, por una parte la liberación de iones debido al aumento de hidróxido de calcio en disolución por la liberación del Ca de la superficie del C3S con un aumento del $\mathrm{pH}$ (siempre muy elevado, llegando incluso a valores de 14), y por otra, una disminución de la presencia de hidróxido cálcico en disolución, por el recubrimiento de los granos de clinker e inicio del proceso de hidratación. Un segundo periodo de latencia en la hidratación se observa entre 6 y 8 horas, en la que se produciría un recubrimiento con los productos de hidratación de los granos de clinker y la penetración hacia el interior de los mismos. A partir de esta etapa se observa un notable incremento de portadores, asociado con la penetración de agua en las partículas y la liberación de iones para la formación de los productos de hidratación.

Es de destacar que en el material con escorias como adicción, desaparece el doble periodo de latencia y activación y lo que es más significativo, se producen diferidamente en el tiempo. Esto puede justificarse porque la presencia de las escorias produce una activación lenta y progresiva en el proceso de hidratación con procesos menos bruscos y más prolongados en el tiempo.

En las figuras 6 a 9, se recoge la evolución de los espectros de difracción de Rayos X de la pasta con y sin adiciones en función del tiempo. Se observa un comportamiento diferenciado para cada uno de los elementos, con la transformación de fases en el tiempo diferente para cada uno de ellos. A modo de ejemplo, en la muestra P-I aparece un fuerte pico asociado a la presencia de yeso $\left(11,9^{\circ}\right)$ que en el caso de las muestras con adiciones prácticamente no se observa. Por su parte, en el rango los picos correspondientes a $23,3^{\circ}$ y 24,5 mantienen una proporción 3/1 en P-I, y esta proporción se reduce a un $4 / 3$ en el caso del P-S. Se observan otras muchas diferencias, pero no es el objeto de este trabajo analizarlas. Para las diferentes regiones con cambios significativos en el tiempo, se ha analizado la evolución de diferentes picos de DRX y se ha analizado éste en función del tiempo. Se pone de manifiesto claramente la aparición y desaparición conjunta de fases, especialmente en etapas iniciales.
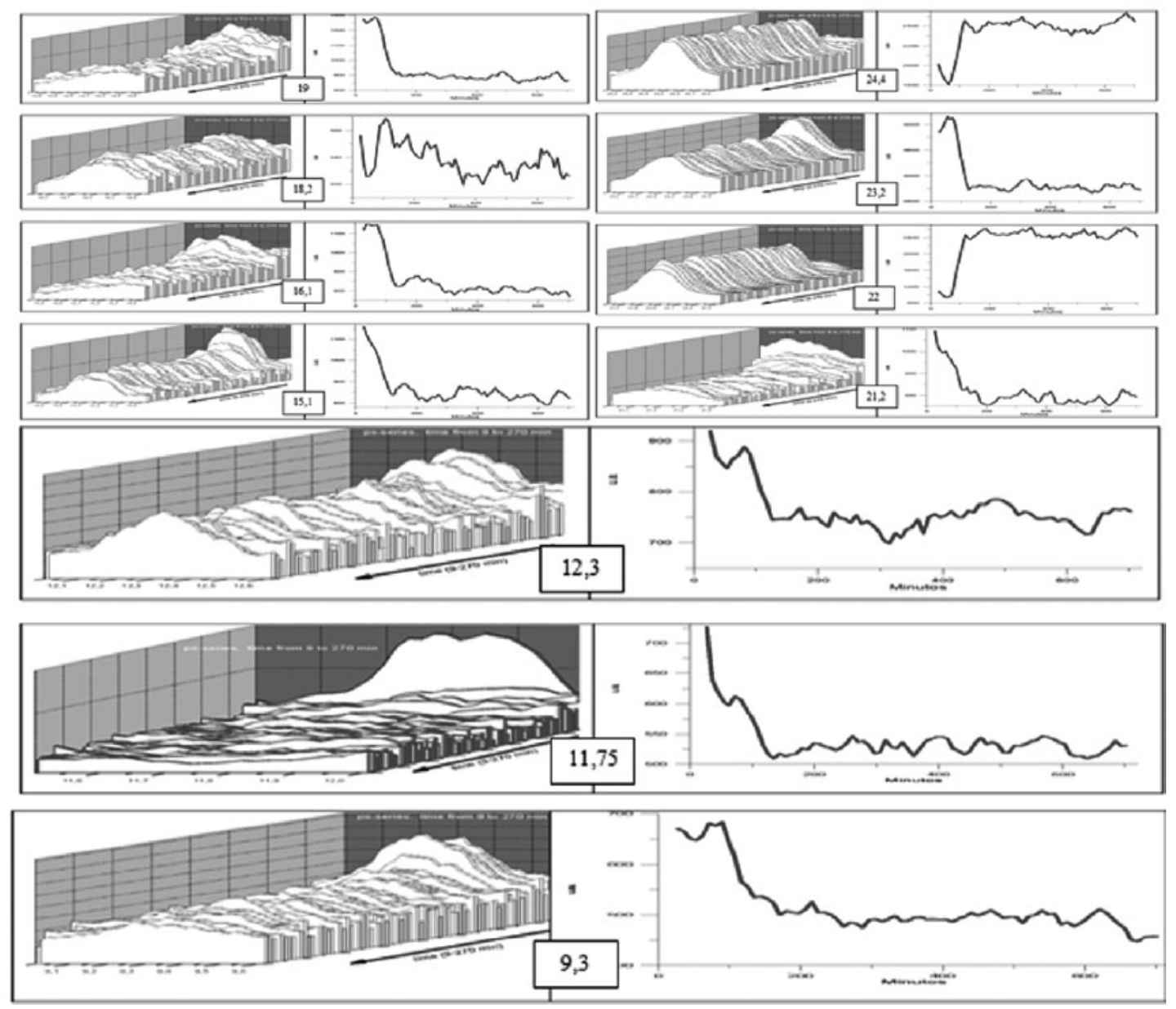

Figura 9. Evolución de diferentes picos de DRX de la muestra P-I durante las doce primeras horas del proceso de hidratación. 
Como se ha indicado anteriormente, el objetivo del estudio de DRX es complementar y verificar la información proporcionada por el estudio mediante espectroscopía de impedancias. Con este fin, en las figuras 6 y 8 se representa la evolución de los espectros de difracción de rayos $X$ respectivamente para las muestras P-I y P-S durante los primeros 740 minutos del proceso de hidratación. En estas figuras se pone de manifiesto la aparición y desaparición de picos asociados a diferentes fases. Para completar esta información, en las figuras 7 y 9, respectivamente para las muestras P-I y P-S, se muestra la evolución con el tiempo del área determinada por diferentes picos de DRX característicos de las fases presentes en el proceso de hidratación.

En todos los casos, hay una concordancia con las variaciones del estado de cristalinidad del material y su comportamiento eléctrico. Si comparamos respectivamente las figuras $7 \mathrm{y}$ 9 con las 3 y 4, observamos que al igual que sucedía con la respuesta eléctrica, que en función de la frecuencia se obtenían resultados diferentes, con la difracción de rayos $\mathrm{X}$, y en concreto para cada fase cristalina tienen una evolución distinta con el tiempo. Los resultados obtenidos permiten suponer que haya una correlación entre ambos tipos de medida, que será objeto de un estudio posterior. Para facilitar la comparación, en la figura 10 se representan conjuntamente los resultados de medidas eléctricas y de DRX.

Si nos centramos en los resultados recogidos en la figura 10, podemos ver que para cada una de las composiciones, hay un buen acuerdo entre los resultados obtenidos por cada una de las técnicas. En el caso de la pasta de referencia P-I, figura 10(A), y con la ayuda de las líneas a trazos, podemos comprobar que las medidas eléctricas ponen de manifiesto los cambios con una ligera anticipación. En todos los casos, aproximadamente a 2 horas se observa un cambio de comportamiento que se mantiene casi hasta las 4 horas, para pasar a otro estado hasta un poco antes de las 6 horas que se prolonga hasta casi las 8 horas que es el momento en el que la respuesta eléctrica presenta un cambio más pronunciado. De nuevo la DRX presenta un cambio de comportamiento asociado a la medida eléctrica que queda más marcado en el caso del DRX16 pero igualmente presente en ambos.
Por su parte, la muestra P-S (figura 10(B)) presenta un comportamiento claramente diferenciado tanto en la respuesta eléctrica como en la de DRX. Para esta composición, existen tres tiempos significativos. En las curvas de DRX, se aprecia un primer cambio notable en la primera hora, que es imperceptible con las medidas eléctricas puesto que corresponde a medidas iniciales. Aproximadamente a dos horas hay un cambio de comportamiento respecto a las etapas iniciales que se pone manifiesto en DRX tanto en la fase que se forma y en la que desaparece así como en las medidas eléctricas. Este proceso se mantiene hasta las 3 horas, tiempo para el que la respuesta eléctrica del material da un máximo de conducción. A partir de este tiempo, con las medidas eléctricas puede apreciarse claramente un aumento de la resistividad del material que se puede asociar a procesos de formación de nuevas estructuras cristalinas, que va asociado con un comportamiento prácticamente uniforme de las fases cristalinas.

\section{CONCLUSIONES:}

- La medida de la impedancia eléctrica, y especialmente a determinadas frecuencias de trabajo, es una herramienta potente para determinar los fenómenos y procesos químicos que tienen lugar en durante las primeras etapas de hidratación de las pastas de cemento.

- El estudio de la respuesta en función de la frecuencia, y comparado los resultados de calor de hidratación, puede concluirse que para frecuencias superiores al $\mathrm{kHz}$, la respuesta determinada proporciona una información muy similar a la del calor de hidratación.

- A frecuencias menores al kHz, aparecen singularidades que pueden asociarse a con los diferentes estadios del proceso de hidratación que tienen lugar en el material. Si bien, para conseguir una información adecuada es necesario establecer un compromiso

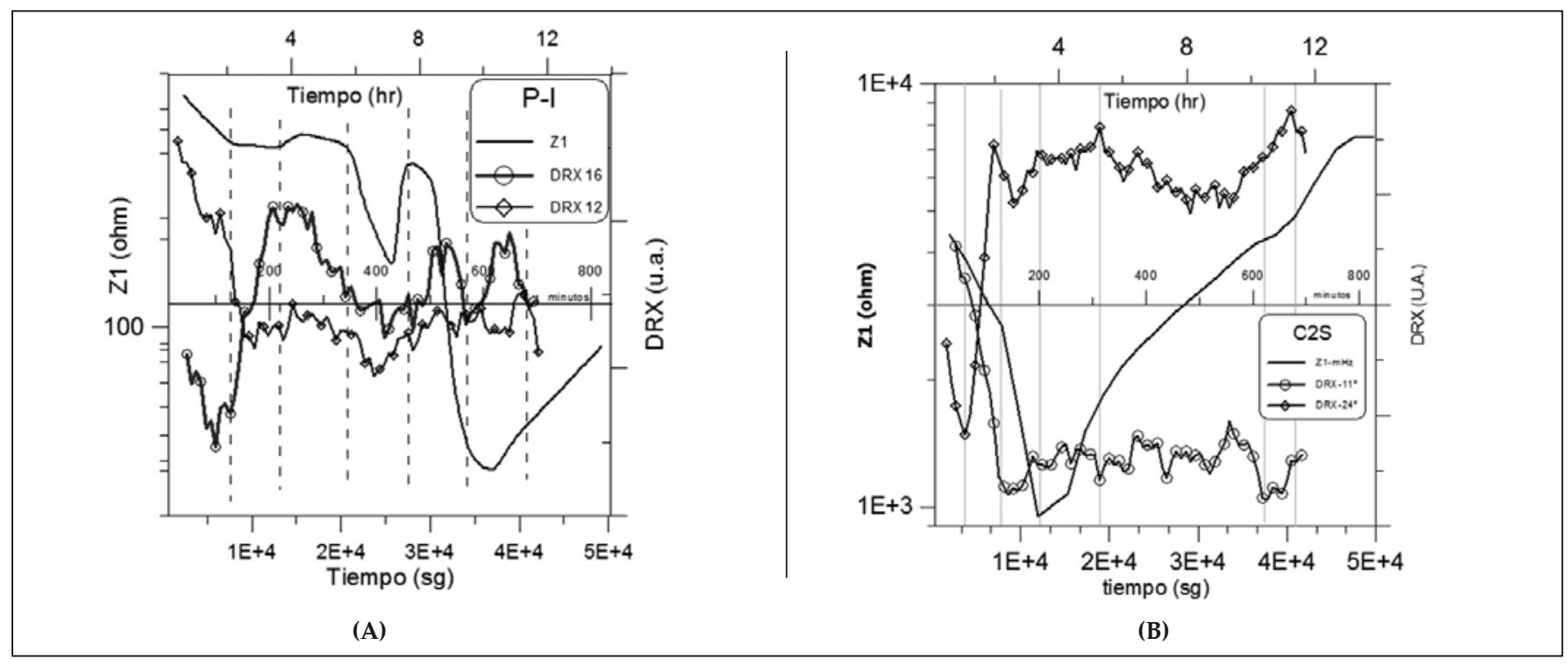

Figura 10. Evolución de diferentes picos de DRX comparados con la evolución de la respuesta eléctrica durante las doce primeras horas del proceso de hidratación, (A) para la pasta de referencia y (B) para la pasta con escorias. 
entre la información que se obtiene y el tiempo para obtener dicha información. Se considera que para una adecuada utilización de este procedimiento de medida, en materiales en base cemento, lo óptimo es realizar las medidas de ciclos consecutivos en el rango de $1 \mathrm{~Hz}$ a $1 \mathrm{kHz}$, haciendo barridos en escala logarítmica y con 5 puntos por década. De esta forma se consiguen secuencias de respuesta en frecuencia con periodos de varios segundos, lo que permite el seguimiento de los procesos que tienen lugar en la hidratación en edades tempranas.

- El estudio de la evolución de los picos de DRX permite relacionar la respuesta eléctrica con la formación/ desaparición de fases cristalinas.

- El estudio realizado pone de manifiesto que añadir escorias a la pasta adelanta el proceso de hidratación, si bien éste es menos intenso que en el cemento sin adición, y se prolonga durante más tiempo.

\section{AGRADECIMIENTOS}

Este trabajo se ha sido posible a la financiación del MEC de España (proyecto MAT2010-21088-C03-03), y de las acciones de consolidación de grupos de investigación 139/Q06 0915110 financiada por la CAM-UPM y AL11-P(I+D)-15 por la UPM

\section{REFERENCIAS}

(1) J.P. Bars, J.P. Camps, J. Debuigne, Exemple et application d'une méthode de mesure d'impédance électrique : Evolution de la conductivité électrique d'une pâte de ciment au cours de sa prise. Mater. Constr. 15(85) 33-37. (1982)

(2) W.J. McCarter, S. Garvin, N. Bouzid, Impedance measurements on cement paste, J. Mater. Sci. Lett. 7 (10) 1056-1057. (1988)

(3) W.J. McCarter, R. Brousseau, The A.C. response of hardened cement paste, Cem. Concr. Res. 20 891-900 (1990).

(4) C.A. Scuderi, T.O. Mason, H.M. Jennings, Impedance spectra of hydrating cement pastes, J. Mater. Sci. 26) 349- 353. (1991

(5) B.J. Christensen, T.O. Mason, H.M. Jennings, Influence of silica fume on the early hydration of Portland cements using impedance spectroscopy, J. Am. Ceram. Soc. 75 (4) 939-945. (1992)

(6) P. Gu, P. Xie, J.J. Beaudoin, R. Brousseau, A.C. impedance spectroscopy: I. A new equivalent circuit model for hydrated Portland cement paste, Cem. Concr. Res. 22 833-840. (1992)

(7) P. Gu, Z. Xu, P. Xie, J.J. Beaudoin, Application of A.C. impedance techniques in studies of porous cementitious materials: I. Influence of solid phase and pore solution on high frequency resistance, Cem. Concr. Res. 23 531- 540 (1993)

(8) C. Andrade, L.Soler, J.deFrutos Dielectric properties of cement paste and its relationship with low water content Bol. Soc. Cer. Vid., 34(5-6), 414-417 (1995).
(9) B.J. Christensen, R.T. Coverdale, R.A. Olson, S.J. Ford, E.J.Garboczi, H.M Jennings, T.O. Mason, Impedance Spectroscopy of Hydrating Cement-Based Materials: Measurement, Interpretation, and Application J. Am. Ceram. Soc. 77 2789. (1994)

(10) H.C. Kim, S.Y. Kim, S.S. Yoon, Electrical Properties of Cement Paste Obtained from Impedance Spectroscopy. J. Mater. Sci. 30 3768. (1995)

(11) P. Xie, P. Gu, Z. Xu, J.J. Beaudoin, Rationalized A.C. Impedance Model for Microstructural Characterization of Hydrating Cement Systems Cem. Concr. Res. 23 359. (1993)

(12) Menendez, E; de Frutos, J; Andrade, C: Internal deterioration of mortars in freeze-thawing: non-destructive evaluation by means of electrical impedance. Adv. Semicond. Mat. 68, 1-11 (2009)

(13) J. de Frutos, L. Soler, C. Andrade. Dielectric properties of concrete paste with low content of free water, Bol. Soc. Esp. Ceram. Vidr., 38(6), 607-610 (1999)

(14) R.A. Olson, B.J. Christensen, R.T. Coverdale, S.J. Ford, G.M. Moss, H.M. Jennings, T.O. Mason, E.J. Garboczi, Interpretation of the impedance spectroscopy of cement paste via computer modelling J. Mater. Sci. 305078. (1995)

(15) D.E. Mcphee, D.C. Sinclair, S.L. Stubbs, Electrical characterization of pore reduced cement by impedance spectroscopy. J. Mater. Sci. Lett. 15 1566; (1996)

(16) D.E. Macphee, D.C. Sinclair, S.L. Cormack, Development of an equivalent circuit model for cement paste from microstructural considerations, J. Am. Ceram. Soc. 80 (11) 2876- 2884. (1997)

(17) H.F.W. Taylor, Cement Chemistry, 2nd ed., Thomas Telford, London, UK, 1997

(18) Menendez, E; De Frutos, J; Andrade, C. Evaluation by means of non destructive techniques to deterioration of mortars due to cycles freeze-thawing. Bol. Soc. Esp. Cer. Vid. 48(5) 223-230 (2009)

(19) G. Dotelli, C.M. Mari, The evolution of cement paste hydration process by impedance spectroscopy, Mater. Sci. Eng. A 303 54-59. (2001)

(20) K. Brantervik, G.A. Niklasson, Circuit models for cement based materials obtained from impedance spectroscopy, Cem. Concr. Res. 21 496- 508 (1991)

(21) G. Song, Equivalent circuit model for AC electrochemical impedance spectroscopy of concrete, Cem. Concr. Res. 30 1723-1730. (2000)

(22) Young-Min Kim, Jong-Heun Lee, Seong-Hyeon Hong. Study of alinite cement hydration by impedance spectroscopy. Cement and Concrete Research 33 299-304. (2003)

(23) Sánchez, X.R. Nóvoa, G. de Vera, M.A. Climent. Microstructural modifications in Portland cement concrete due to forced ionic migration tests. Study by impedance spectroscopy. Cement and Concrete Research, Volume 38(7), 1015-1025. (2008)

(24) Koleva, D.A.; de Wit, J.H.W.; van Breugel, K., Veleva, L.P.; van Westing, E.; Copuroglu, O.; Fraaig, A.L.A. Correlation of microstructure, electrical properties and electrochemical phenomena in reinforced mortar. Breakdown to multi-phase interface structure. Part I: Microstructural observations and electrical properties. Materials Characterization 59. Pages 801-816 (2008).

(25) EN 197-1:2000. Cement. Part 1. Composition, specifications and conformity criteria of the common cements. (2000).

(26) EN 196:4:2005. Method of cement tests. Physical test. Determination of hydration heat by semi-adiabatic calorimetry (Method of Langabant calorimetry) (2005)

(27) De Frutos, J; Calzada, M.L.; Menéndez E. A study of ionic defects in modified lead titanate ceramics J. Mat Sci, 30 (11) 2866-2872 (1995)

(28) de Frutos, J.; Gonzalez, F.; Villafuerte-Castrejon, M.E.; Gonzalez, A.M.; Menendez, E.; Jimenez, F.J. Study of Miscibility in Mixed Systems . Int. Ferroelectrics. 101 80-88 (2008)

(29) P. Kumar Mehta. "Concrete Structure, Properties and Materials". Ed. PrenticeHall, Inc., Englewood Cliffs, New Jersey (USA). ISBN 0-13-167115-4. (1986)

(30) W. Chen y H.J.H. Brouwers. "The reaction of slag in cement: theory and computer modeling". Journal of Material Science 42 (2). pp. 428-464 (2007).

(31) Menendez, E; de Frutos, J, Ion monitoring solutions in cements using electrical spectroscopy. Rev. Mex. Phys. 55(1) 76-80 (2009) 


\section{Introducción a los esmaltes cerámicos}

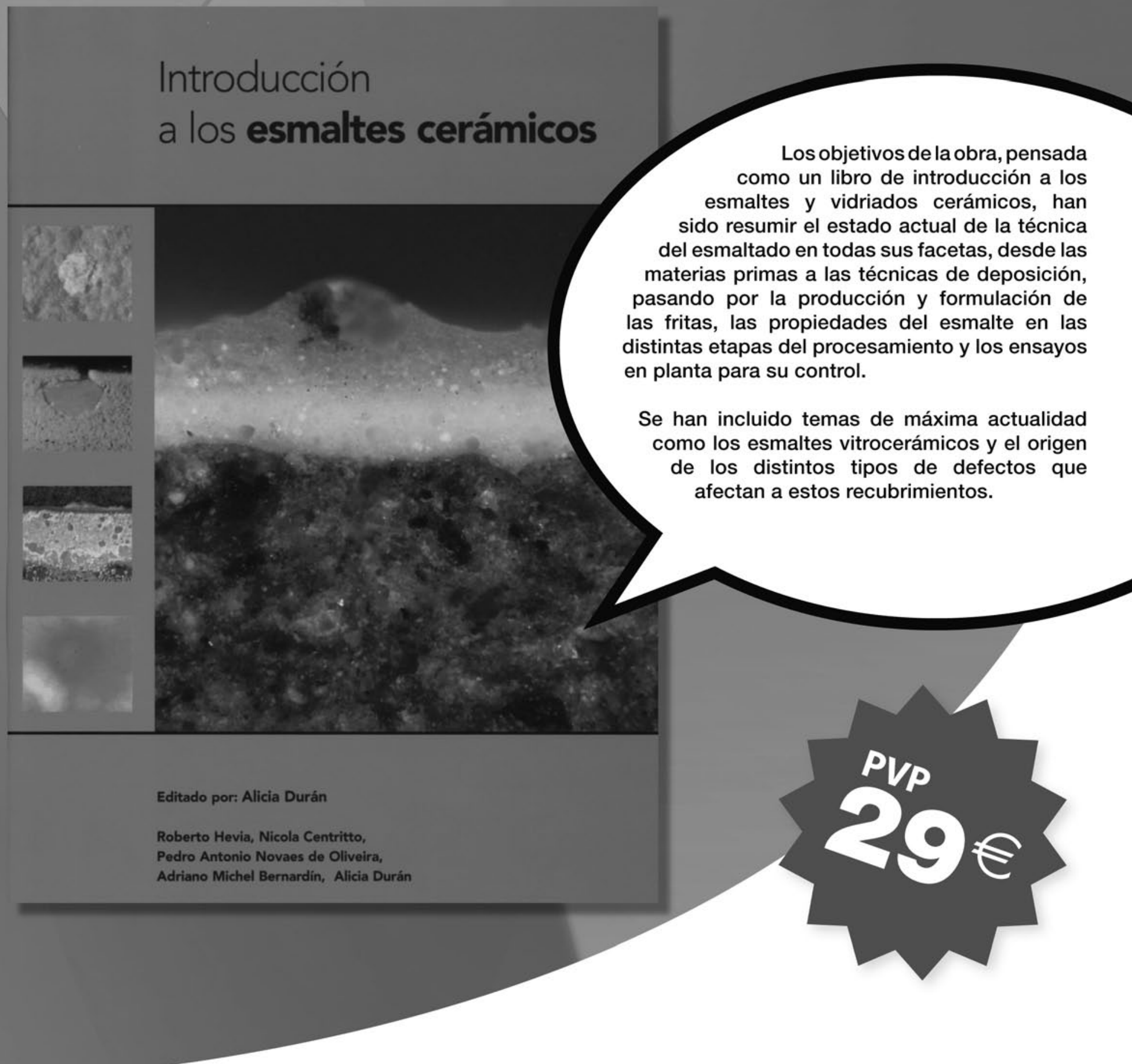

los distintos tipos de defectos que 